\title{
Urine levels of HMGB1 in Systemic Lupus Erythematosus patients with and without renal manifestations
}

\author{
Deena A Abdulahad', Johanna Westra ${ }^{1 *}$, Johannes Bijzet ${ }^{2}$, Sebastian Dolff ${ }^{1,5}$, Marcory C van Dijk ${ }^{3}$, \\ Pieter C Limburg ${ }^{2}$, Cees GM Kallenberg ${ }^{1}$ and Marc Bij ${ }^{4}$
}

\begin{abstract}
Introduction: Lupus nephritis (LN) is a severe and frequent manifestation of systemic lupus erythematosus (SLE). Its pathogenesis has not been fully elucidated but immune complexes are considered to contribute to the inflammatory pathology in LN. High Mobility Group Box 1 (HMGB1) is a nuclear non-histone protein which is secreted from different types of cells during activation and/or cell death and may act as a pro-inflammatory mediator, alone or as part of DNAcontaining immune complexes in SLE. Urinary excretion of HMGB1 might reflect renal inflammatory injury. To assess whether urinary HMGB1 reflects renal inflammation we determined serum levels of HMGB1 simultaneously with its urinary levels in SLE patients with and without LN in comparison to healthy controls (HC). We also analyzed urinary HMGB1 levels in relation with clinical and serological disease activity.
\end{abstract}

Methods: The study population consisted of 69 SLE patients and 17 HC. Twenty-one patients had biopsy proven active $L N$, 15 patients had a history of LN without current activity, and 33 patients had non-renal SLE. Serum and urine levels of HMGB1 were both measured by western blotting. Clinical and serological parameters were assessed according to routine procedures. In 17 patients with active LN a parallel analysis was performed on the expression of HMGB1 in renal biopsies.

Results: Serum and urinary levels of HMGB1 were significantly increased in patients with active LN compared to patients without active LN and HC. Similarly, renal tissue of active LN patients showed strong expression of HMGB1 at cytoplasmic and extracellular sites suggesting active release of HMGB1. Serum and urinary levels in patients without active LN were also significantly higher compared to HC. Urinary HMGB1 levels correlated with SLEDAl, and showed a negative correlation with complement C3 and C4.

Conclusion: Levels of HMGB1 in urine of SLE patients, in particular in those with active LN, are increased and correlate with SLEDAI scores. Renal tissue of LN patients shows increased release of nuclear HMGB1 compared to control renal tissue. HMGB1, although at lower levels, is, however, also present in the urine of patients without active LN. These data suggest that urinary HMGB1 might reflect both local renal inflammation as well as systemic inflammation.

\section{Introduction}

Systemic lupus erythematosus (SLE) is a prototypic systemic autoimmune disease characterized by a wide array of autoantibodies, mainly against nuclear components. Autoantibody production is associated with various clinical manifestations and among these manifestations, renal involvement, that is, lupus nephritis (LN), is the most

\footnotetext{
* Correspondence: Johanna.Westra@med.umcg.nl

'Department of Rheumatology and Clinical Immunology, University Medical Center Groningen, University of Groningen, PO Box 30.001, 9700RB

Groningen, The Netherlands

Full list of author information is available at the end of the article
}

serious clinical problem predicting morbidity and mortality $[1,2]$. The mechanisms underlying the pathogenesis of LN are not fully elucidated. However, LN has often been considered an inflammatory disease resulting from deposition of preformed immune complexes or binding of autoantibodies to antigens localized to glomeruli, socalled in situ complex formation [3-5]. Among the many antibodies potentially participating in the formation of immune complexes, antibodies against DNA are the hallmark of SLE. Recently, it has been shown that these DNA-containing immune complexes constitute among 
others, high mobility group box 1 (HMGB1), which has been suggested to be involved in binding of these immune complexes to renal tissue and initiate renal injury [6].

HMGB1 is a nuclear DNA-binding protein that resides inside the nucleus and can be released to the extracellular space under specific conditions [7,8]. Whereas HMGB1 is actively released from lipopolysaccharide (LPS), TNF-, and IL-1 activated monocytes and macrophages, its release also occurs passively during the late phase of apoptosis as well as during necrosis [7,9,10]. Extracellularly, HMGB1 acts as an alarmin involved in inflammatory reactions through binding to its functional receptors, that is the receptor of advanced glycation end products (RAGE) and toll-like receptors (TLR)-2, -4, and -9 [11-14].

There is accumulating evidence that HMGB1 contributes to the pathogenesis of inflammatory and autoimmune diseases, especially SLE [15-17]. This is related to the fact that apoptotic cells accumulate in SLE and are the main source of autoantigens, including HMGB1 $[7,18]$. We and others showed that serum levels of HMGB1 are elevated in SLE patients and correlate with SLE disease activity score and, inversely, with levels of the complement components $C 3$ and $\mathrm{C} 4$. Moreover, we could demonstrate that serum levels of HMGB1, in particular, were increased in SLE patients with active renal disease and correlated with proteinuria [15]. The origin of the increased serum levels of HMGB1 is not known, and HMGB1 could possibly result from release from damaged and/or inflamed renal tissue. As such, HMGB1 could appear in the urine during (active) LN. In this study, we hypothesize that urinary excretion of HMGB1 reflects renal inflammatory injury in SLE. We investigated this by measuring HMGB1 levels in the urine of SLE patients and correlating this to clinical and biochemical measures of renal and systemic disease activity.

\section{Materials and methods \\ Patients}

Sixty-nine patients (nine male, sixty female), median age 41 (range 16 to 81 ) years, who fulfilled at least four of the American College of Rheumatology (ACR) revised criteria for the diagnosis of SLE, and seventeen healthy controls (HC) (two male, fifteen female), median age 26 (range 20 to 59) years were enrolled in the study. The study was approved by the Medical Ethical Committee of the University Medical Center Groningen, University of Groningen, the Netherlands, and all patients and $\mathrm{HC}$ gave informed consent. Clinical data were obtained from all patients and the study was conducted according to the ethical guidelines of our institution in accord with the Declaration of Helsinki. Among the patients, 21 had biopsy-proven active LN, 15 had a history of LN without current activity, and 33 had non-renal SLE (Table 1). Disease activity at the time of blood sampling was assessed by the SLE disease activity index (SLEDAI). Currently active LN ( $\mathrm{n}=21$ ) was defined as proliferative glomerulonephritis (class III or IV) in a parallel-obtained renal biopsy $(n=17)$ or as the presence of an active urinary sediment representing glomerular injury in a patient with biopsy-proven LN before. Blood and urine was obtained when patients were admitted, at a maximum of 3 days before a renal biopsy was taken. Further characteristics of the patients are summarized in Table 1.

\section{Materials}

Serum and urine samples were collected from patients and HC. Patients' urine samples that were nitrite-positive urine samples on a dip stick test or demonstrated evidence of bacterial contamination in the sediment were excluded. Levels of anti-dsDNA, C-reactive protein $(\mathrm{CRP})$, creatinine $(\mathrm{Cr})$, and complement factors $(\mathrm{C} 3, \mathrm{C} 4)$ in sera, and $24 \mathrm{hr}$ proteinuria and urine $\mathrm{Cr}$ levels were determined by routine techniques.

Paraffin-embedded sections of renal biopsy specimens obtained from 17 patients with active LN were included in the present study. Renal tissues from three unaffected parts of the kidneys of patients with renal cell carcinoma were used as controls.

\section{Western blot for serum and urine HMGB1}

Serum and urine samples from SLE patients and HC were collected and analyzed for HMGB1 by Western Blotting as described previously. Western blot was chosen because commercially available ELISA kits are not suitable for SLE sera, due to interference of autoantibodies (15). Also these kits are not recommended for urine samples. Reproducibility was calculated over the positive control in eight blots performed on different days and coefficient of variation was $22.1 \%$.

Urine samples were concentrated between 30 and 300 times using Vivaspin 15R (Sartorius Stedim Biotech, Gottingen, Germany). Serum and urine samples from SLE patients and $\mathrm{HC}$ were diluted in SDS buffer $(0.063 \mathrm{M}$ Tris. $\mathrm{HCl}$ pH 6.8, 2\% SDS, 10\% glycerol, 0.015\% BromePhenol Blue, and 5\% ß-mercaptoethanol) and heated at $98^{\circ} \mathrm{C}$ for five minutes. The volume of urine loaded to the gel was corrected for the concentration factor. Next, proteins were resolved on 12.5\% SDS-PAGE gel (Criterion gel BioRad, Veenendaal, The Netherlands) and transferred to polyvinylidene fluoride membrane (Millipore, Amsterdam, The Netherlands) followed by incubation with anti-HMGB1 mouse monoclonal antibody (1:250; R\&D Systems, Abingdon, UK). Detection was done with polyclonal goat anti-mouse IgG labelled with IRDye800 (1: 5000; LI-COR Biotechnology, Westburg, Leusden, the Netherlands). Blots were scanned with Odyssey infrared Imaging System (LI-COR Biotechnology) and then analyzed with the Odyssey software (version 2.1). A standard 
Table 1 Demographic, clinical and laboratory data of systemic lupus erythematosus (SLE) patients at the time of the study

\begin{tabular}{|c|c|c|c|}
\hline & \multirow[t]{2}{*}{ Active LN } & \multicolumn{2}{|l|}{ No active LN } \\
\hline & & History of LN & No history of LN \\
\hline Total number & 21 & 15 & 33 \\
\hline Male/female, number & $4 / 17$ & $3 / 12$ & $2 / 31$ \\
\hline Age, years, median (range) & 38 (16 to 53 ) & 46 (18 to 61$)$ & 48 (20 to 81$)$ \\
\hline SLEDAI, mean \pm SD & $13.7 \pm 3.7^{* * *,+++}$ & $2.7 \pm 1.7$ & $2.8 \pm 2.7$ \\
\hline Anti-dsDNA antibody, IU/ml, median (range) & $140(3 \text { to } 1,000)^{* *,+}$ & 19 (3 to 432$)$ & 20.5 (3 to 1000$)$ \\
\hline C3, g/l, median (range) & $0.55(0.27 \text { to } 1.13)^{* * *,++}$ & 0.93 (0.43 to 1.48$)$ & $0.97(0.31$ to 11.4$)$ \\
\hline C4 (g/l), median (range) & $0.08(0.03-0.3)^{*,+}$ & $0.16(0.06$ to 0.3$)$ & $0.12(0.02$ to 1.04$)$ \\
\hline CRP, mg/l, median (range) & $5(5$ to 121$)$ & 5 ( 5 to 21 ) & 5 (5 to 33$)$ \\
\hline eGFR, $\mathrm{ml} / \mathrm{min}$ & $72(24$ to 142$)$ & $81(45$ to 145$)$ & 89 (31 to 140$)$ \\
\hline Serum creatinine, $\mu \mathrm{mol} / \mathrm{l}$, median (range) & 75 (46 to 406$)$ & $71(51$ to 126$)$ & $62(47$ to 141$)$ \\
\hline 24-hr proteinuria, $\mathrm{g} / 24 \mathrm{hr}$ & $1.5(0.3 \text { to } 8.4)^{* * *,+++}$ & $0.3(0.2 \text { to } 2.5)^{\# \# \# ~ \# ~}$ & $0.0(0.0$ to 0.1$)$ \\
\hline Urine creatinine, $\mathrm{mmol} / \mathrm{l}$, median (range) & $7.6(0.6$ to 21.3$)$ & $4.3(1.6$ to 15.7$)$ & $6.2(0.7$ to 14.9$)$ \\
\hline With/without treatment, number & $12 / 9$ & $13 / 2$ & $21 / 12$ \\
\hline Patients using prednisone, number (\%), dose, mg/day, median (range) & $\begin{array}{l}10(47.6 \%) \\
7.5(2.5 \text { to } 60)\end{array}$ & $\begin{array}{l}10(66.6 \%) \\
10(5 \text { to } 60)\end{array}$ & $\begin{array}{l}8(24.2 \%) \\
5(1.25 \text { to } 10)\end{array}$ \\
\hline $\begin{array}{l}\text { Patients taking hydroxychloroquine, number (\%), dose, mg/day, } \\
\text { median (range) }\end{array}$ & $\begin{array}{l}8(38 \%) \\
350(200 \text { to } 600)\end{array}$ & $\begin{array}{l}2(13.3 \%) \\
400(400)\end{array}$ & $\begin{array}{l}14(42.2 \%) \\
400(200 \text { to } 600)\end{array}$ \\
\hline Patients using azathioprine, number (\%), dose, mg/day, median (range) & $\begin{array}{l}3(14.3 \%) \\
100(75 \text { to } 150)\end{array}$ & $\begin{array}{l}7(46.6 \%) \\
75(50 \text { to } 150)\end{array}$ & $\begin{array}{l}6(18.2 \%) \\
125(75 \text { to } 150)\end{array}$ \\
\hline
\end{tabular}

${ }^{+}$Indicates difference between active lupus nephritis (LN) patients and patients with no active LN but with history of LN $\left({ }^{+} P \leq 0.05,{ }^{++} P \leq 0.005,{ }^{+++} P \leq 0.0005\right)$. *Indicates difference between active and inactive SLE patients ( $\left.{ }^{*} P=0.05,{ }^{*} P \leq 0.005,{ }^{* *} P \leq 0.0005\right)$. ${ }^{*}$ Indicates difference between patients without active LN but with history of LN and inactive SLE patients with no history of LN ( $\left.{ }^{\#} P=0.05,{ }^{\#} P \leq 0.005,{ }^{\# \#} P \leq 0.0005\right)$. Anti-dsDNA: anti-double stranded DNA; C3: Complement 3; C4 Complement 4; CRP: C-reactive protein; eGFR: estimated glomerular filtration rate; SLEDAl: systemic lupus erythematosus disease activity index.

sample was obtained from lyzed human keratinocyte $\mathrm{HaCaT}$ cells and included in each blot as an internal control. In each blot, levels of HMGB1 were expressed as values of fluorescence intensity and were normalized against the standard sample. Urinary HMGB1 was expressed as HMGB1/Cr ratio (intensity units/mmol/l) to correct for differences in dilution.

\section{Analysis of renal biopsies}

Biopsies concomitantly taken with serum and urine samples were processed. All biopsies were reviewed and classified by an experienced nephropathologist (MCvD) according to the revised criteria for LN [19]. The activity index (AI) and chronicity index (CI) were calculated for each specimen with maximum scores of 24 for the AI and 12 for the CI [19]. Histological data are shown in Table 2.

\section{Immunohistochemical staining of renal biopsies}

Kidney sections $(4 \mu \mathrm{m})$ were used for all staining experiments. Sections were deparaffinised. Next, antigen retrieval and endogenous peroxidase blocking was performed. Slides were incubated with rabbit anti-HMGB1 antibody (Abcam, Cambridge, UK), goat anti-RAGE antibody (AbD Serotec, Dusseldorf, Germany), rabbit anti-TLR2 antibody (Abcam), and rabbit anti-TLR-4 antibody (Abcam). Subsequently, slides were incubated with HRP-labelled secondary antibodies (DakoCytomation, Glostrup, Denmark).
Next, slides were incubated in diaminobenzidine solution and counterstained with hematoxylin. Using ImageScope software (Aperio Technologies, Vista, CA, USA) morphometry was performed on entire renal sections stained with antibodies against RAGE, TLR2 and TLR4.

\section{Analysis of immunohistochemical staining Evaluation of HMGB1 staining}

The stained slides were coded and analyzed independently by two observers (DA and JW) in a blinded fashion. Cellular distribution of HMGB1 was determined in the kidney by counting one hundred nuclei in three brightfield pictures and scoring both HMGB1-positive (brown) and HMGB1-negative (blue) nuclei. Results are expressed as the percentage of negative cells.

\section{Evaluation of TLR2, TLR4 and RAGE staining}

Expression of TLR 2, TLR4 and RAGE was analyzed on entire renal tissue sections of active LN patients and controls stained with antibodies against TLR2, TLR4 and RAGE.

Glass slides were digitally scanned with $20 \times$ objective using Aperio ScanScope (Aperio Technologies). The images were quantitatively analyzed and enhanced using the computer software system ImageScope (Aperio Technologies) for histological examination. The positive pixel count algorithm was used to quantify the amount of a specific stain present in a scanned image. Colour (range of 
Table 2 Histological data of renal biopsies from seventeen systemic lupus erythematosus (SLE) patients with active renal disease and three controls

\begin{tabular}{|c|c|c|c|c|c|}
\hline Number & Sex & ISN class & Activity index(AI) & Chronicity index(CI) & HMGB1-negative nuclei in renal tissues, \% \\
\hline 1 & $\mathrm{~F}$ & IV & 10 & 3 & 35.0 \\
\hline 2 & $\mathrm{~F}$ & III & 3 & 7 & 40.0 \\
\hline 3 & $\mathrm{~F}$ & III & 2 & 0 & 25.0 \\
\hline 4 & $\mathrm{~F}$ & IV & 8 & 0 & 20.4 \\
\hline 5 & $\mathrm{~F}$ & III & 3 & 0 & 28.1 \\
\hline 6 & $\mathrm{~F}$ & III & 1 & 4 & 29.4 \\
\hline 7 & $\mathrm{~F}$ & IV & 5 & 0 & 37.2 \\
\hline 8 & $\mathrm{~F}$ & IV & 3 & 2 & 24 \\
\hline 9 & $\mathrm{~F}$ & IV & 5 & 3 & 38 \\
\hline 10 & $F$ & III & 4 & 3 & 26 \\
\hline 11 & $\mathrm{~F}$ & IV & 3 & 0 & 42 \\
\hline 12 & $\mathrm{~F}$ & III & 2 & 0 & 0.7 \\
\hline 13 & $\mathrm{~F}$ & III & 7 & 2 & 31.1 \\
\hline 14 & M & III & 3 & 0 & 12 \\
\hline 15 & M & na & 1 & 0 & 19.1 \\
\hline 16 & $\mathrm{~F}$ & IV & 6 & 2 & 26.3 \\
\hline 17 & $\mathrm{~F}$ & IV & 8 & 3 & 26.3 \\
\hline 18 Control & F & - & - & - & 0 \\
\hline 19 Control & $\mathrm{F}$ & - & - & - & 0 \\
\hline 20 Control & $F$ & - & - & - & 0 \\
\hline
\end{tabular}

M: male; F: female; ISN: International Society of Nephrology; HMGB1: high mobility group box 1; na: not assessed.

hues and saturation) and intensity were specified. For pixels that satisfied the colour specification, the algorithm counted the number and intensity sum in each intensity range. Positive colour class was divided into three intensity ranges producing four categories of staining, namely strong, medium, weakly positive, and negative staining. Each pixel that stained was put into one of the four categories and total pixel counts were reported, along with intensity, for each category. The percentage of positivity was calculated by using the average of positive intensities divided by the total numbers of positively and negatively stained pixels.

\section{Statistical analysis}

Data are presented as median (range) unless stated otherwise. Statistical analysis was performed by using the statistical package Graph Pad Prism, version 3.02 (Graph Pad software Inc., San Diego, CA, USA). A student Mann-Whitney test was performed for comparison of different groups as appropriate. Spearman rank correlation was used to assess correlations. A $P$-value $<0.05$ was considered significant.

\section{Results}

\section{Serum and urine HMGB1 levels}

Serum HMGB1 levels in active LN patients were significantly increased (intensity 57 , range 11 to 350 ) compared to $\mathrm{HC}$ (intensity 6,1 to 38 ) and also compared to patients without active LN (intensity 16, 2 to 92) (Figure 1A).
Patients without active LN, but with a history of renal involvement had relatively higher levels of serum HMGB1 (intensity 20, 2 to 80) compared to those without renal involvement (intensity 13, 2 to 92), but this difference was not statistically significant.

Representative blots of urine measurements of HMGB1 are shown in Figure 1D. In lanes 3, 5, 7, 9, 11, 13 and 15, patients' samples were run (even lanes were left empty), while in lanes 2 and 18 the positive control is shown. In each blot a urine sample of a $\mathrm{HC}$ was run in lane 17, whereas a molecular weight marker was run in lane 1. Urinary HMGB1 levels were significantly increased in patients with active LN (intensity 54, 4 to 300) compared to $\mathrm{HC}$ (intensity 0,0 to 4 ) and to patients with no active $\mathrm{LN}$ (intensity 4, 0 to 300) (Figure $1 \mathrm{~B}$ ). In patients with no active LN but having a history of LN, levels of urinary HMGB1 were increased (intensity 20, 0 to 300), but not significantly compared to patients without a history of LN (1, 0 to 87 ) (Figure 1B). To correct for dilution, urinary levels were expressed as $\mathrm{HMGB1/Cr}$ ratios. In Figure $1 \mathrm{C}$ these ratios are shown in the patient groups, and also here a significant difference was seen between patients with active $\mathrm{LN}$ and patients without active $\mathrm{LN}$.

\section{Correlation of urine HMGB1 levels with clinical and serological findings}

Since urine levels of HMGB1 were increased in SLE patients, particularly in patients with active LN, we investigated whether urine levels of HMGB1 were 


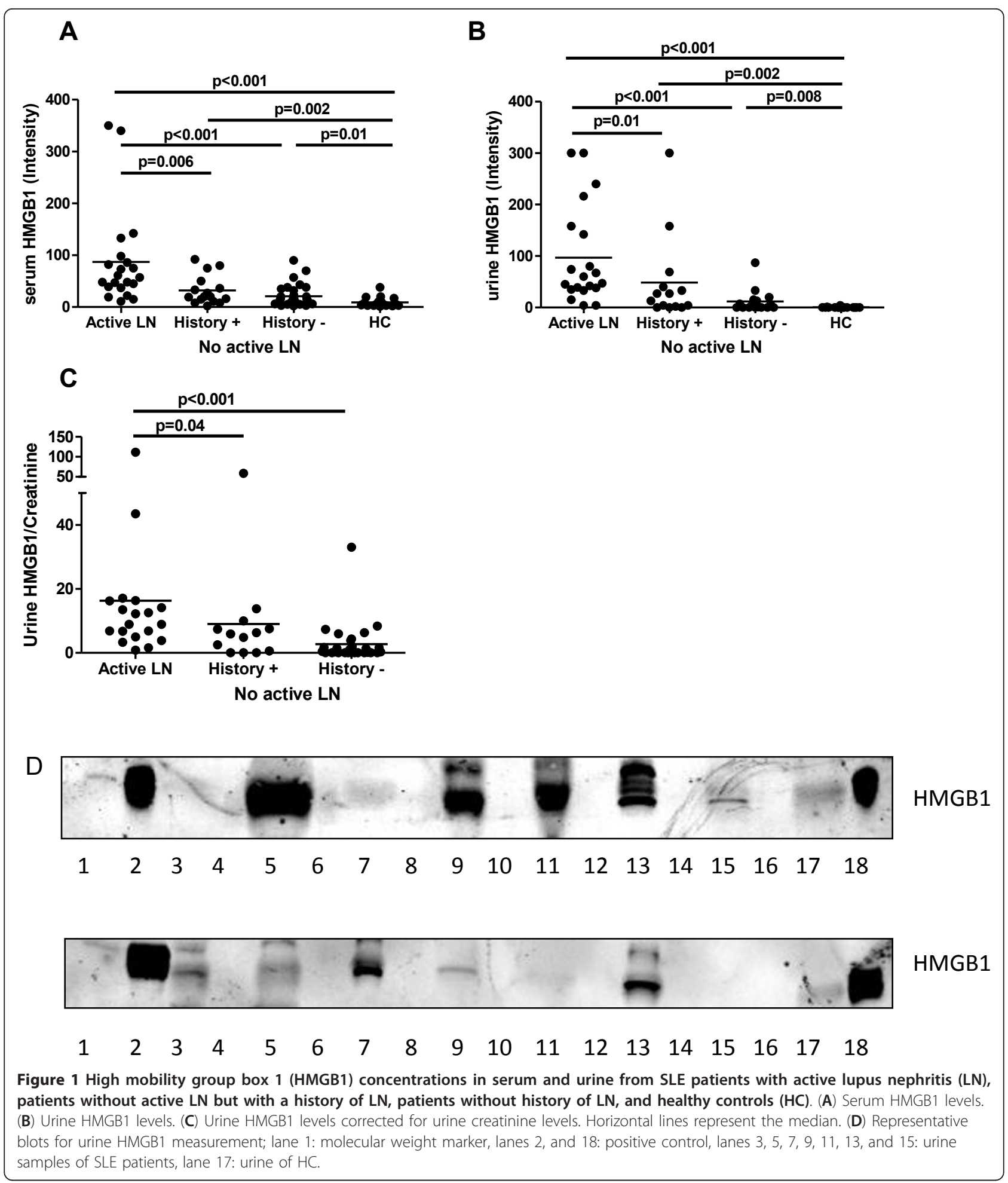

related to clinical and serological parameters of disease activity, and might thus be used as an additional marker for assessment of disease activity, in particular renal activity. We observed a positive correlation between urine HMGB1/Cr ratios and SLEDAI $(\mathrm{r}=0.54, P<$
0.0001) (Figure 2A). Also, a significant negative correlation was observed between levels of $\mathrm{C} 3$ and urinary HMGB1/Cr ratios $(r=-0.38, P=0.008)$ (Figure $2 \mathrm{~B}$ ). A positive correlation was observed between urine HMGB1/Cr ratios and proteinuria $(\mathrm{r}=0.57, P<0.0001)$ 


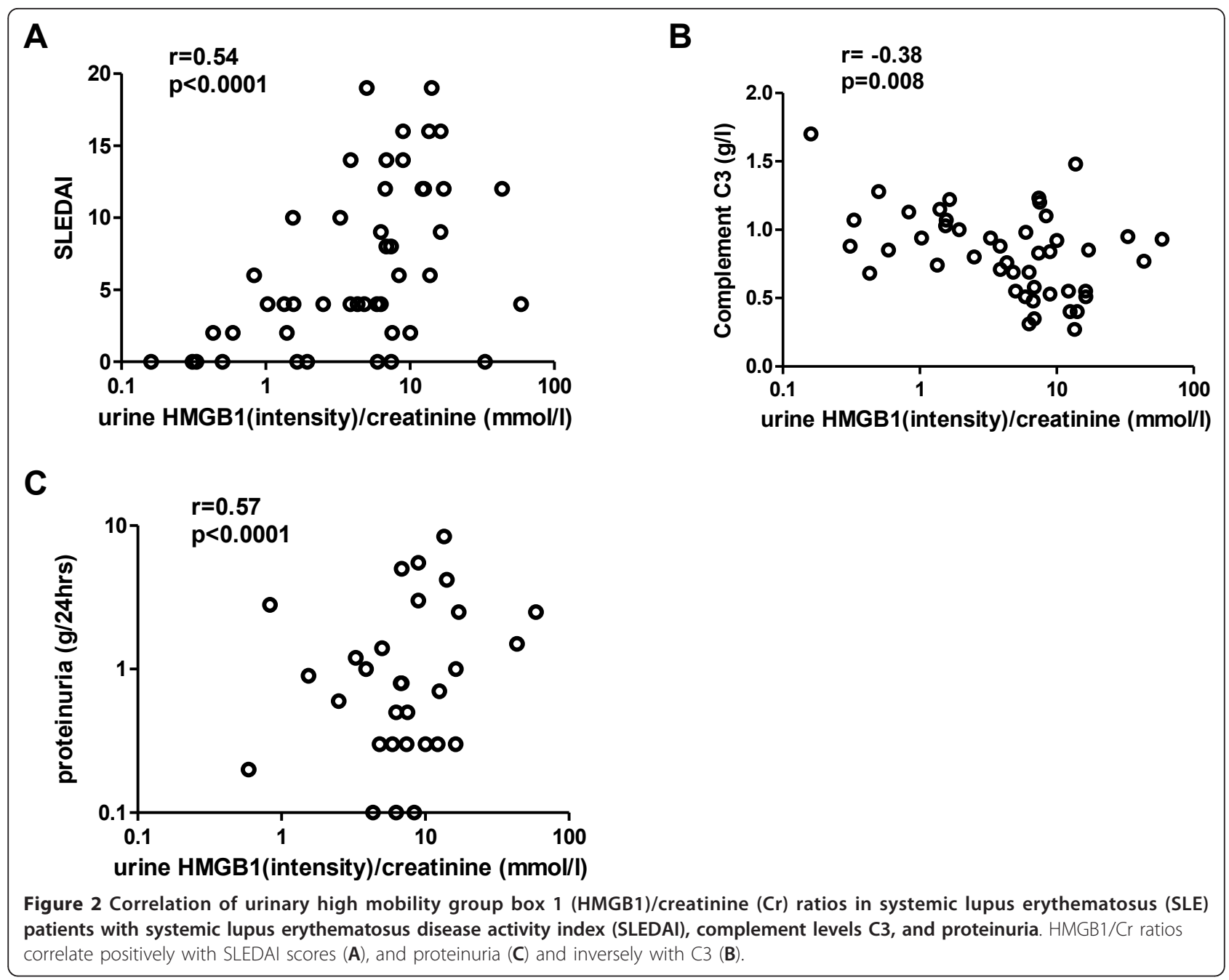

(Figure 2C). Next we assessed the relation between serum HMGB1 and proteinuria in order to analyze whether urinary HMGB1 passively results from proteinuria. In this group of patients, serum HMGB1 levels were not correlated to proteinuria (Figure 3A). However, urinary HMGB1 and serum HMGB1 levels were positively correlated $(\mathrm{r}=0.28, P=0.056)$ (Figure $3 \mathrm{~B}$ ).

\section{Release of nuclear HMGB1 in the kidney}

To assess the role of HMGB1 in renal inflammation or damage we assessed the presence and localization of HMGB1 in renal biopsies taken from patients with active $\mathrm{LN}$ in comparison to control renal tissue. In control renal tissue, HMGB1 was mainly present in the nuclei with minor cytoplasmic staining in the tubuli (Figure 4C) and in the glomeruli (Figure 4D). In biopsy sections from patients with LN, HMGB1 was no longer confined to the nuclei only, but was mainly expressed in the cytoplasm and the extracellular space (Figure 4E and 4F). In these patients, significant percentages of HMGB1 negative nuclei (resulting from HMGB1 release) were present. The percentage of HMGB1-negative nuclei was counted and data are summarized in Table 2. Although the glomerular structures of patients with active LN showed significant extracellular staining of HMGB1, only a few HMGB1-negative nuclei were detected in glomeruli. No correlation was found between the percentages of HMGB1 negative nuclei in renal tissue and levels of urinary HMGB1. We further assessed the association between the percentage of HMGB1 negative nuclei in the kidney, and histological findings such as activity index (AI) and chronicity index (CI) and renal function parameters (eGFR, creatinine and proteinuria). No correlation was found between histological scores and HMGB1 negative nuclei in the kidney (data not shown). Although no correlation was found between HMGB1 negative nuclei in the kidney and renal parameters (creatinine and proteinuria), there was a trend toward a negative correlation with estimated glomerular filtration rate (eGFR) $(r=$ $-0.51, P=0.05)$. 


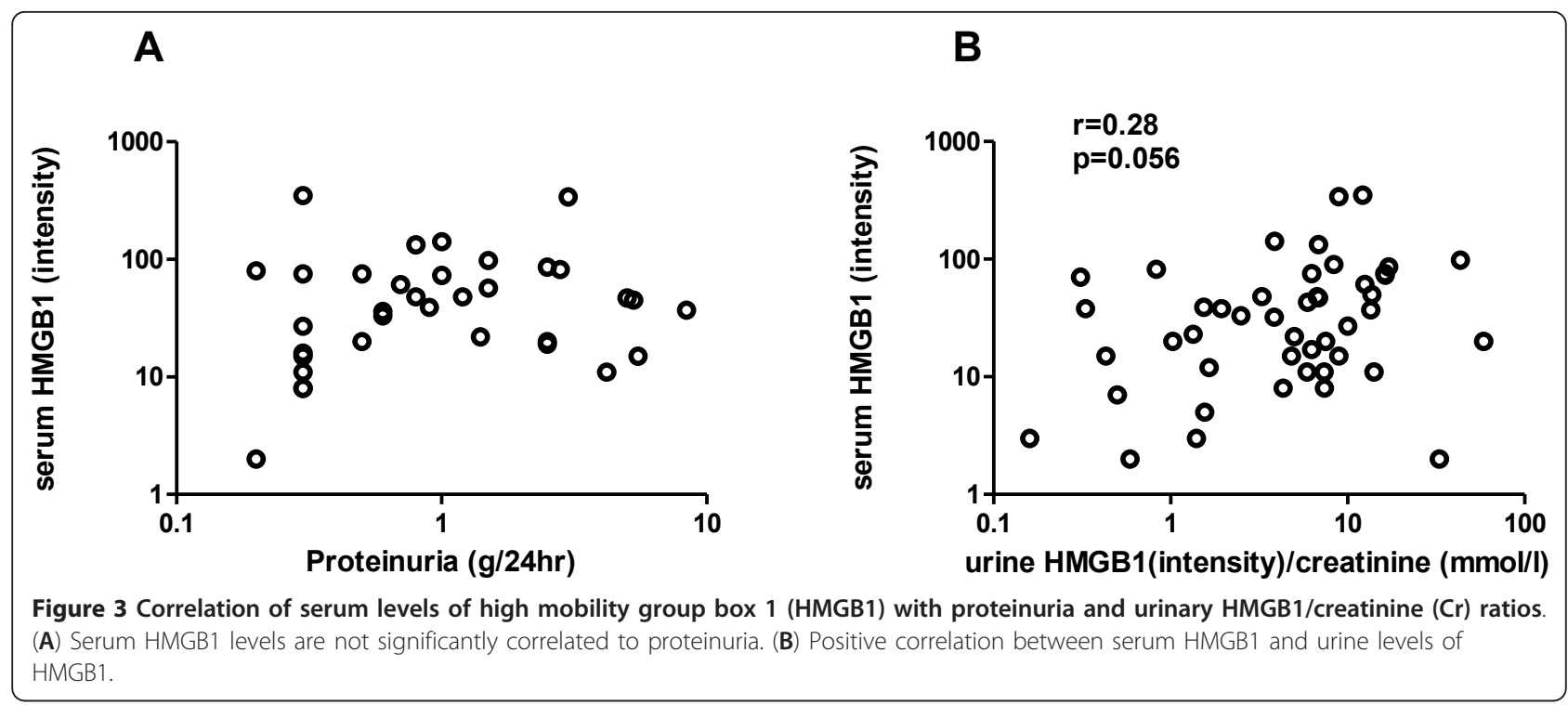

A

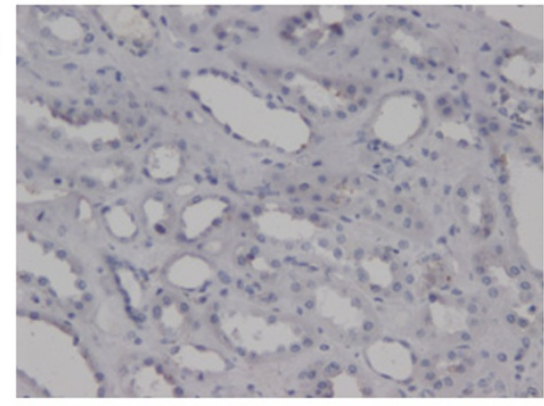

$\mathrm{C}$

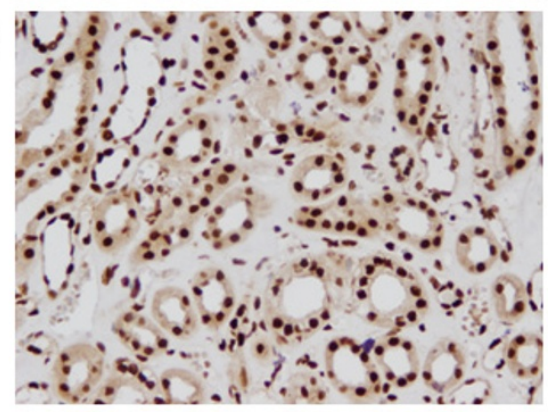

E

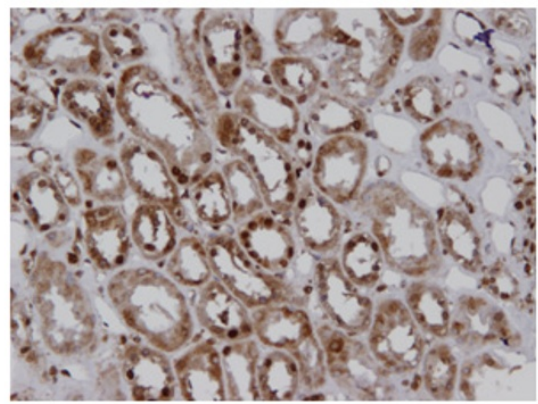

B

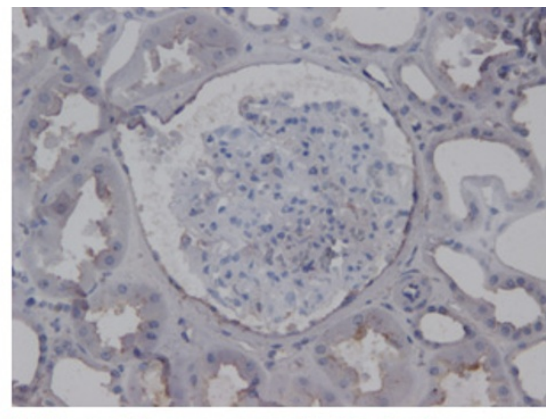

$\mathrm{D}$

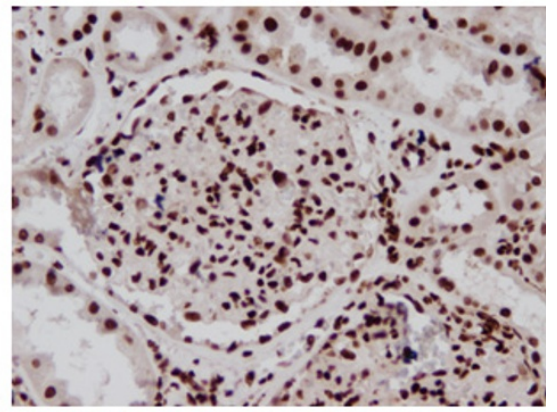

$\mathrm{F}$ :

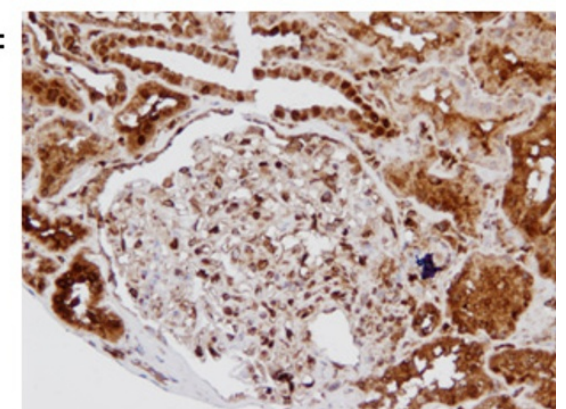

Figure 4 Expression of high mobility group box 1 (HMGB1) in renal tissue of active lupus nephritis (LN) patients and controls Representative immunohistochemical staining of HMGB1 in a renal biopsy from an active LN patient $(\mathbf{E}, \mathbf{F})$ and control renal tissue $(\mathbf{C}, \mathbf{D})$. Isotype staining in control tissue $(\mathbf{A}, \mathbf{B})$. Renal tissue of the active LN patient shows a higher percentage of HMGB1-negative nuclei in tubular cells (E) and strong cytoplasmic and extracellular staining for HMGB1 in tubuli and glomeruli (F). Biopsy taken from normal renal tissue shows expression of HMGB1 mainly inside nuclei (C,D). 
Expression of HMGB1 receptors (TLR2, TLR4 and RAGE) in the kidney of LN patients and controls

As expression and release of HMGB1 was increased in the renal biopsies from $\mathrm{LN}$ patients, we further analyzed the expression of HMGB1 receptors, that is, TLR2, TLR4 and RAGE, in these biopsies as well as in control renal tissue. Control renal tissue showed relatively weak expression of TLR2, TLR4 and RAGE with 14\% (12 to 16\%) for TLR2, $14 \%$ (12 to $16 \%$ ) for TLR4 and $0.2 \%$ (0.004 to $0.500 \%$ ) for RAGE. In contrast, strong expression of TLR2, TLR4 and RAGE was observed in renal biopsies from patients with active LN compared to control renal tissue, with 28.7 (24.7 to $50.0 \%$ ), $P<0.008$, for TLR2; $28.8 \%$ (20 to $40 \%$ ), $P<$ 0.008 , for TLR and 29\% (17 to 40\%), $P<0.008$, for RAGE. Expression of TLR2, TLR4 and RAGE in active LN patients was mainly cytoplasmic and localized in glomeruli as wells as in tubular cells (Figure 5). Expression of various receptors was not associated with the percentage of
HMGB1-negative nuclei in renal biopsies from patients with active LN, SLEDAI and renal function parameters.

\section{Discussion}

In the present study we demonstrated for the first time that HMGB1 levels are significantly increased in the urine of SLE patients especially in those with active proliferative LN. Moreover, urinary levels of HMGB1 were correlated with disease activity scores, and reversely with levels of complement C3.

LN is a serious manifestation in patients with SLE that can progress to end-stage renal failure. Despite extensive studies, the pathogenesis of LN has not been fully clarified. Recently, the role of HMGB1 has been demonstrated in autoimmunity and chronic inflammatory diseases, especially those with renal manifestations such as antineutrophil cytoplasmic antibody (ANCA)-associated vasculitis, chronic kidney disease (CKD) and SLE [15,17,20-25].
A

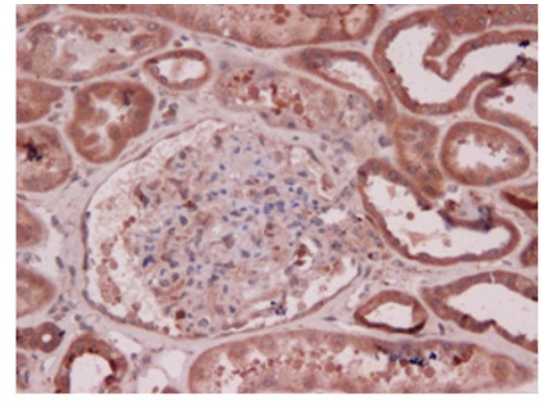

C

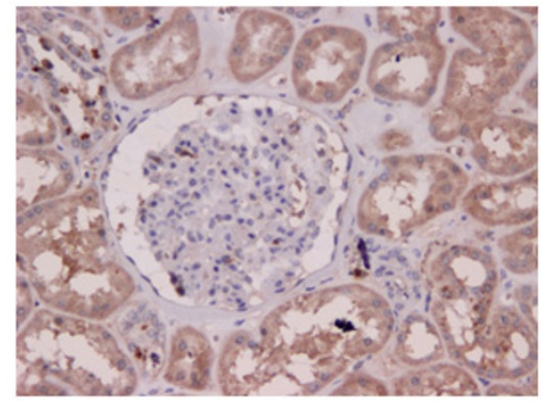

E

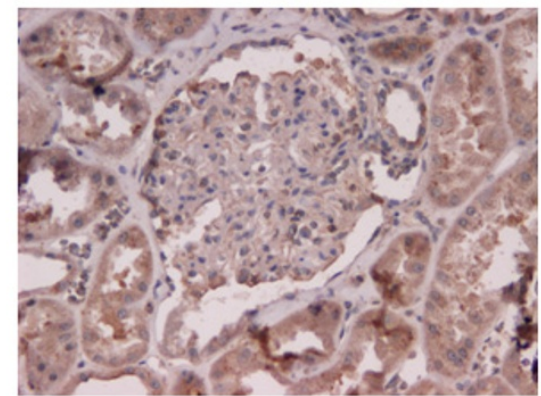

B

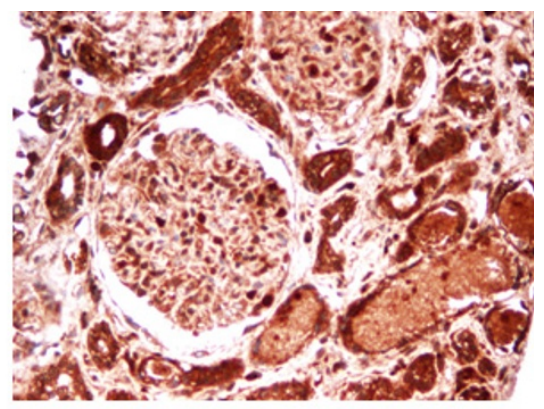

D

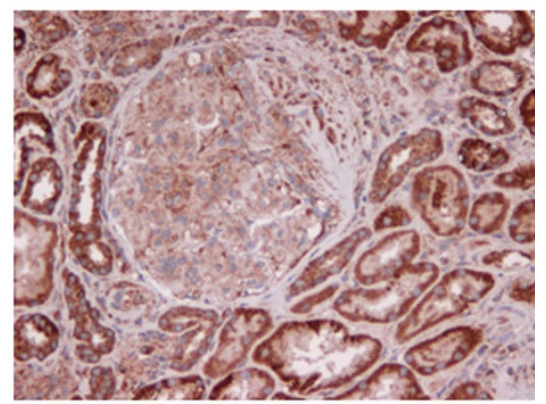

$\mathrm{F}$

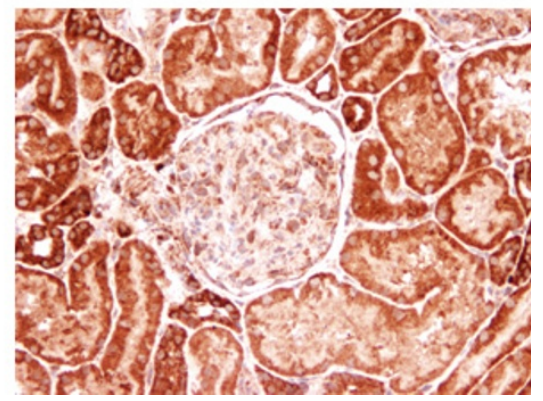

Figure 5 Expression of high mobility group box 1 (HMGB1) receptors in a renal biopsy from a patient with active lupus nephritis (LN) and in control renal tissue. Representative staining for RAGE, TLR2 and TLR4 in active LN $(\mathbf{D}, \mathbf{E}, \mathbf{F})$ and control renal tissue $(\mathbf{A}, \mathbf{B}, \mathbf{C})$, respectively. Renal tissue of an active LN patient shows strong cytoplasmic/extracellular expression for HMGB1 receptors in tubular cells, interstitial infiltrates, and glomeruli in comparison to control tissue. 
However, until now no study has been conducted to evaluate urinary levels of HMGB1 as a reflection of renal inflammation. Thus, in the current study we investigated urine levels of HMGB1, in comparison to serum levels, in SLE patients with and without renal involvement. In accordance with our previous study, serum levels of HMGB1 were significantly increased in active LN patients compared to patients without active renal disease and controls [15]. Similarly, urinary levels of HMGB1 were increased in patients with active LN. Of note, urinary HMGB1 levels were also detectable, but at a lower level, in patients without active LN, but not in HC. Detectable amounts of HMGB1 in the urine of patients without active LN might be explained in two ways. A possibly ongoing low-grade renal inflammatory activity could contribute to the release of HMGB1. Indeed, Zickert et al. showed that extracellular expression of HMGB1 is detectable in renal biopsies taken from quiescent SLE patients at follow up [26]. Furthermore, some patients with SLE may have negative urine findings despite histological evidence of lupus nephritis [27]. Second, increased levels of serum HMGB1 might lead to urinary excretion of HMGB1, particularly in patients with a history of LN and slight persisting proteinuria. HMGB1 has been recognized as a new autoantigen and as an important inflammatory mediator in SLE as exemplified by increased serum levels and presence of antibodies against this protein [15-17]. Both HMGB1 and anti-HMGB1 antibodies have been shown to be associated with SLE disease activity, decreased complement levels, and proteinuria [15]. In our present study however, we did not find a positive correlation of HMGB1 serum levels with proteinuria. Now a large group of patients with newly diagnosed active LN without induction therapy was included in contrast to our previous study. This may explain the discrepancy in findings between both studies. Nevertheless, the absence of a correlation in the present study is not fully explained.

Interestingly, in the current study urinary HMGB1 levels were correlated with SLEDAI, particularly in patients with active LN. Furthermore, urinary levels of HMGB1 were inversely correlated with complement levels. We were not able to determine prospectively if urine levels of HMGB1 can be used for monitoring renal disease activity in patients with active LN. Nevertheless, increased urine levels of HMGB1 might indicate that HMGB1 is an important inflammatory mediator and that urinary HMGB1 might be an additional biomarker for assessment of renal disease activity in SLE. However, more studies are needed to ascertain that urinary levels of HMGB1 are a clinically useful marker.

The exact events leading to glomerular inflammation and damage are still not known. Anti-dsDNA or antinucleosome antibodies, present in immune complexes, have been suggested as important players. It has been shown that HMGB1 is an important component of these circulating DNA-containing immune complexes [6]. HMGB1 is responsible for immune-inflammatory activity and for binding of DNA-containing immune complexes, containing anti-dsDNA and/or anti-HMGB1 antibodies, to renal tissue, causing injury. However, the origin of HMGB1, whether it is produced outside the kidney or locally within the inflamed kidney, is still unresolved. In the current study, the percentage of HMGB1-negative nuclei, probably resulting from HMGB1 release, was higher in renal tissue of patients with active LN compared to control renal tissue, suggesting active release of HMGB1 in pro-inflammatory processes within the kidney. Our results are in line with the findings of Qing et al. [6]. In their study, specific data on the characteristics of their LN patients were not mentioned, and levels of HMGB1 in serum were not detectable. Also, urinary levels of HMGB1 were not measured. We were not able to definitely identify the cells releasing HMGB1. Release of HMGB1 could result from infiltrating cells as indicated by immunohistochemical staining, but could also result from either activation or cell death of constitutive renal tissue. Also, we cannot exclude the possibility that at least some of urinary HMGB1 might emerge from systemic inflammation. This might explain the lack of correlation between urinary HMGB1 and HMGB1 released from nuclei in the kidney. Further support for the role of HMGB1 in renal inflammation comes from the increased expression of the HMGB1 receptors TLR2, TLR4 and RAGE in the renal tissue of LN patients. To the best of our knowledge, this is the first study describing expression of the functional receptors (TLR2, TLR4 and RAGE) of HMGB1 in lupus nephritis. The low intrinsic expression of these receptors in control tissue suggests that TLR2, TLR4, and RAGE are all part of the innate immune system in the kidney. The intense and altered expression of TLR2, TLR4, and RAGE in patients with active LN, however, could reflect a role of these receptors in the development and severity of lupus nephritis. In combination, all these data indicate that HMGB1 might be an important player in the pathogenesis of $\mathrm{LN}$ and, possibly, a therapeutic target. Indeed, Mao et al. demonstrated that targeting HMGB1 by monoclonal antibodies in a murine adenovirus-accelerated SLE model inhibited the development of proteinuria and proved protective [28]. Clinical studies targeting HMGB1, however, have not been performed.

The present study provides evidence that HMGB1 expression is increased not only in the sera but also in the urine of active LN patients as well as at the site of local renal inflammation. Urine levels of HMGB1 could reflect both local and systemic inflammation. Urinary biomarkers might be more relevant than serum biomarkers because they may directly reflect renal pathology. 
However, further studies are needed to evaluate the clinical significance of measuring urinary HMGB1 and its value as a biomarker in lupus patients with renal involvement.

\section{Conclusions}

The present study demonstrated increase in urine HMGB1 levels in SLE patients, in particular in those with active LN. Increase in urine HMGB1 levels correlated to SLE disease activity index (SLEDAI). Accordingly, renal tissue of LN patients showed increase in nuclear HMGB1 release compared to control tissue. Furthermore, expression of TLR2, TLR4 and RAGE was increased in a renal biopsy from active LN patients. Together, we suggest that HMGB1 may play an important role in renal pathology in SLE patients.

\section{Abbreviations \\ ACR: American College of Rheumatology; Al: activity index; ANCA antineutrophil cytoplasmic antibody-associated vasculitis; anti-dsDNA: anti- double stranded DNA; C3: Complement 3; C4 Complement 4; Cl: chronicity index; CKD: chronic kidney disease; Cr: creatinine; CRP: C-reactive protein; eGFR: estimated glomerular filtration rate; ELISA: enzyme-linked immunosorbant assay; HMGB1: high mobility group box 1; LN: lupus nephritis; LPS: lipopolysaccharide; RAGE: receptor for advanced glycation end products; SLE: systemic lupus erythematosus; SLEDAI: systemic lupus erythematosus disease activity index; TLRs: toll like receptors. \\ Acknowledgements \\ The study was supported by a research grant (GUIDE graduate school number 71812) from University Medical Center Groningen, Groningen, The Netherlands and by a research grant from the Deutsche Forschungs Gemeinschaft (DO 1419/1-1)}

\section{Author details}

'Department of Rheumatology and Clinical Immunology, University Medical Center Groningen, University of Groningen, PO Box 30.001, 9700RB Groningen, The Netherlands. ${ }^{2}$ Pathology and Laboratory Medicine, University Medical Center Groningen, University of Groningen, PO Box 30.001, 9700RB Groningen, The Netherlands. ${ }^{3}$ Department of Pathology, University Medical Center Groningen, University of Groningen, PO Box 30.001, 9700RB Groningen, The Netherlands. ${ }^{4}$ Department of Internal Medicine and Rheumatology, Martini Hospital, PO Box 30.033, 9700RM Groningen, The Netherlands. ${ }^{5}$ Department of Nephrology, University Hospital Essen, University Duisburg-Essen, 47057 Duisburg, Germany.

\section{Authors' contributions}

DAA, JW and MB designed the study and DAA and JB did the HMGB1 measurements. SD collected patients' materials and MCVD performed biopsy preparation and classification. DAA, JW, JB, PCL, CGMK and MB were involved in interpretation of the results, while DAA, JW, MB and CGMK critically prepared the manuscript. All authors read and approved the final manuscript.

\section{Competing interests}

The authors declare that they have no competing interests.

Received: 27 April 2012 Revised: 12 July 2012

Accepted: 14 August 2012 Published: 14 August 2012

\section{References}

1. Austin HA, Boumpas DT, Vaughan EM, Balow JE: Predicting renal outcomes in severe lupus nephritis: contributions of clinical and histologic data. Kidney Int 1994, 45:544-550.
2. Bono L, Cameron JS, Hicks JA: The very long-term prognosis and complications of lupus nephritis and its treatment. QJM 1999, 92:211-218.

3. Berden JH: Lupus nephritis. Kidney Int 1997, 52:538-558.

4. Manson JJ, Ma A, Rogers P, Mason LJ, Berden JH, van der Vlag J, D'Cruz DP, Isenberg DA, Rahman A: Relationship between anti-dsDNA, antinucleosome and anti-alpha-actinin antibodies and markers of renal disease in patients with lupus nephritis: a prospective longitudinal study. Arthritis Res Ther 2009, 11:R154.

5. Sasaki T, Muryoi T, Hatakeyama A, Suzuki M, Sato H, Seino J, Saito T, Yoshinaga $\mathrm{K}$ : Circulating anti-DNA immune complexes in active lupus nephritis. Am J Med 1991, 91:355-362.

6. Qing X, Pitashny M, Thomas DB, Barrat FJ, Hogarth MP, Putterman C: Pathogenic anti-DNA antibodies modulate gene expression in mesangial cells: involvement of HMGB1 in anti-DNA antibody-induced renal injury. Immunol Lett 2008, 121:61-73.

7. Bell CW, Jiang W, Reich CF, Pisetsky DS: The extracellular release of HMGB1 during apoptotic cell death. Am J Physiol Cell Physiol 2006, 291 C1318-C1325.

8. Landsman D, Bustin M: A signature for the HMG-1 box DNA-binding proteins. Bioessays 1993, 15:539-546.

9. Wang $H$, Vishnubhakat JM, Bloom O, Zhang M, Ombrellino M, Sama A, Tracey KJ: Proinflammatory cytokines (tumor necrosis factor and interleukin 1) stimulate release of high mobility group protein-1 by pituicytes. Surgery 1999, 126:389-392.

10. Gardella S, Andrei C, Ferrera D, Lotti LV, Torrisi MR, Bianchi ME, Rubartelli A: The nuclear protein HMGB1 is secreted by monocytes via a nonclassical, vesicle-mediated secretory pathway. EMBO Rep 2002, 3:995-1001.

11. Harris HE, Raucci $A$ : Alarmin(g) news about danger: workshop on innate danger signals and HMGB1. EMBO Rep 2006, 7:774-778.

12. Kokkola R, Andersson A, Mullins G, Ostberg T, Treutiger CJ, Arnold B, Nawroth P, Andersson U, Harris RA, Harris HE: RAGE is the major receptor for the proinflammatory activity of HMGB1 in rodent macrophages. Scand J Immunol 2005, 61:1-9.

13. Tian J, Avalos AM, Mao SY, Chen B, Senthil K, Wu H, Parroche P, Drabic S, Golenbock D, Sirois C, Hua J, An LL, Audoly L, La Rosa G, Bierhaus A, Naworth P, Marshak-Rothstein A, Crow MK, Fitzgerald KA, Latz E, Kiener PA, Coyle AJ: Toll-like receptor 9-dependent activation by DNA-containing immune complexes is mediated by HMGB1 and RAGE. Nat Immunol 2007, 8:487-496.

14. Yu M, Wang H, Ding A, Golenbock DT, Latz E, Czura CJ, Fenton MJ, Tracey KJ, Yang H: HMGB1 signals through toll-like receptor (TLR) 4 and TLR2. Shock 2006, 26:174-179.

15. Abdulahad DA, Westra J, Bijzet J, Limburg PC, Kallenberg CG, Bijl M: High mobility group box 1 (HMGB1) and anti-HMGB1 antibodies and their relation to disease characteristics in systemic lupus erythematosus. Arthritis Res Ther 2011, 13:R71.

16. Hayashi A, Nagafuchi H, Ito I, Hirota K, Yoshida M, Ozaki S: Lupus antibodies to the HMGB1 chromosomal protein: epitope mapping and association with disease activity. Mod Rheumatol 2009, 19:283-292.

17. Li J, Xie H, Wen T, Liu H, Zhu W, Chen X: Expression of High Mobility Group Box Chromosomal Protein 1 and Its Modulating Effects on Downstream Cytokines in Systemic Lupus Erythematosus. J Rheumatol 2010, 37:766-775.

18. Casciola-Rosen LA, Anhalt G, Rosen A: Autoantigens targeted in systemic lupus erythematosus are clustered in two populations of surface structures on apoptotic keratinocytes. J Exp Med 1994, 179:1317-1330.

19. Weening JJ, D'Agati VD, Schwartz MM, Seshan SV, Alpers CE, Appel GB, Balow JE, Bruijn JA, Cook T, Ferrario F, Fogo AB, Ginzler EM, Hebert L, Hill G, Hill $P$, Jennette JC, Kong NC, Lesavre P, Lockshin M, Looi LM, Makino H, Moura LA, Nagata M: The classification of glomerulonephritis in systemic lupus erythematosus revisited. J Am Soc Nephrol 2004, 15:241-250.

20. Bruchfeld A, Qureshi AR, Lindholm B, Barany P, Yang L, Stenvinkel P, Tracey KJ: High Mobility Group Box Protein-1 correlates with renal function in chronic kidney disease (CKD). Mol Med 2008, 14:109-115.

21. Bruchfeld A, Wendt M, Bratt J, Qureshi AR, Chavan S, Tracey KJ, Palmblad K, Gunnarsson I: High-mobility group box-1 protein (HMGB1) is increased in antineutrophilic cytoplasmatic antibody (ANCA)-associated vasculitis with renal manifestations. Mol Med 2011, 17:29-35.

22. Kim J, Sohn E, Kim CS, Jo K, Kim JS: The role of high-mobility group box-1 protein in the development of diabetic nephropathy. Am J Nephrol 2011, 33:524-529. 
23. Ma CY, Jiao YL, Zhang J, Yang QR, Zhang ZF, Shen YJ, Chen ZJ, Zhao YR: Elevated plasma level of HMGB1 is associated with disease activity and combined alterations with IFN-alpha and TNF-alpha in systemic lupus erythematosus. Rheumatol Int 2012, 32:395-402.

24. Oyama Y, Hashiguchi T, Taniguchi N, Tancharoen S, Uchimura T, Biswas KK, Kawahara K, Nitanda T, Umekita Y, Lotz M, Maruyama I: High-mobility group box-1 protein promotes granulomatous nephritis in adenineinduced nephropathy. Lab Invest 2010, 90:853-866.

25. Sato F, Maruyama S, Hayashi H, Sakamoto I, Yamada S, Uchimura T, Morita Y, Ito Y, Yuzawa Y, Maruyama I, Matsuo S: High mobility group box chromosomal protein 1 in patients with renal diseases. Nephron Clin Pract 2008, 108:c194-c201.

26. Zickert A, Palmblad K, Sundelin B, Chavan S, Tracey K, Bruchfeld A, Gunnarsson I: Renal expression and serum levels of high mobility group box 1 protein in lupus nephritis. Arthritis Res Ther 2012, 14:R36.

27. Wada Y, Ito S, Ueno M, Nakano M, Arakawa M, Gejyo F: Renal outcome and predictors of clinical renal involvement in patients with silent lupus nephritis. Nephron Clin Pract 2004, 98:c105-111.

28. Mao SY, Xu Y, Zhang J, Schifferli K, An LL, Leininger J, Kiener P, Wu H, Coyle AJ, Audoly LP: Antagonizing HMGB1 inhibits proteinuria in a murine model of lupus like disease. (abstract). J Immunol 2008, 178:131-124

doi:10.1186/ar4015

Cite this article as: Abdulahad et al:: Urine levels of HMGB1 in Systemic Lupus Erythematosus patients with and without renal manifestations. Arthritis Research \& Therapy 2012 14:R184.

\section{Submit your next manuscript to BioMed Central and take full advantage of:}

- Convenient online submission

- Thorough peer review

- No space constraints or color figure charges

- Immediate publication on acceptance

- Inclusion in PubMed, CAS, Scopus and Google Scholar

- Research which is freely available for redistribution

Submit your manuscript at www.biomedcentral.com/submit 